\title{
Cost Analysis of a Home-Based Virtual Reality Rehabilitation to Improve Upper Limb Function in Stroke Survivors
}

\author{
Mirella Veras ${ }^{1}$, Jennifer Stewart ${ }^{2}$, Raywat Deonandan ${ }^{3}$, José Carlos Tatmatsu-Rocha ${ }^{4}$, \\ Johanne Higgins ${ }^{5}$, Lise Poissant ${ }^{5} \&$ Dahlia Kairy $^{5}$ \\ ${ }^{1}$ Centre de Recherche Interdisciplinaire en Réadaptation du Montréal métropolitain (CRIR), University of \\ Montreal, Canada; Kings Regional Rehabilitation Centre, Canada \\ ${ }^{2}$ School of Public Policy and Administration, Carleton University, Ottawa, Canada \\ ${ }^{3}$ Interdisciplinary School of Health Sciences, University of Ottawa, Canada \\ ${ }^{4}$ Medicine School, Physical Therapy Department, Federal University of Ceara, Fortaleza, Ceara, Brazil \\ ${ }^{5}$ Centre de recherche interdisciplinaire en réadaptation du Montréal métropolitain (CRIR), Institut universitaire \\ sur la réadaptation en déficience physique de Montréal (IURDPM) du Centre intégré universitaire de santé et de \\ services sociaux du Centre-Sud-de-l'Ile-de-Montréal, École de réadaptation, Université de Montréal, Montreal, \\ Canada \\ Correspondence: Mirella Veras, Centre de recherche interdisciplinaire en réadaptation du Montréal métropolitain \\ (CRIR), QC H3S 1M9, Montreal, Canada. E-mail: mvera025@uottawa.ca
}

\author{
Received: December 25, 2019 Accepted: January 17, 2020 Online Published: January 30, 2020 \\ doi:10.5539/gjhs.v12n2p98 \\ URL: https://doi.org/10.5539/gjhs.v12n2p98
}

\begin{abstract}
Loss of arm function occurs in up to $85 \%$ of stroke survivors. Home-based telerehabilitation is a viable approach for upper limb training post-stroke when rehabilitation services are not available. Method: A costing analysis of a telerehabilitation program was conducted under several scenarios, alongside a single-blind two-arm randomized controlled trial with participants randomly allocated to control $(\mathrm{N}=25)$ or intervention group $(\mathrm{N}=26)$. Detailed analysis of the cost for two different scenarios for providing telerehabilitation were conducted. The fixed costs of the telerehabilitation are an important determinant of the total costs of the program. The detailed breakdown of the costs allows for costs of future proposed telerehabilitation programs to be easily estimated. The costs analysis found that a program supplying all required technology costs between CAD $\$ 475$ per patient and CAD $\$ 482$ per patient, while a program supplying only a camera would have total costs between CAD $\$ 242$ per patient and \$245 per patient. The findings of this study support the potential implementation of telerehabilitation for stroke survivors for improving accessibility to rehabilitation services. This cost-analysis study will facilitate the implementation and future research on cost-effectiveness of such interventions.
\end{abstract}

Keywords: telerehabilitation, stroke recovery, neurorehabilitation, remote rehabilitation assistance, telemedicine, telehealth

\section{Introduction}

Stroke is a leading cause of mortality and the most important cause of severe adult long-term disability (Salter K, 2007). It is considered a global public health problem due to the resulting severe permanent impairments, functional limitations in daily living activities, reduced leisure and occupational activities, and mental problems such as risk of depression (Baumann, Le Bihan, Chau, \& Chau, 2014). Loss of arm function occurs in up to $85 \%$ of stroke survivors (Nichols-Larsen, Clark, Zeringue, Greenspan, \& Blanton, 2005), with a significant impact on quality of life (Kitzmüller, Asplund, \& Häggström, 2014). Although most rehabilitation treatments focus on the first three months after stroke, studies show that stroke has a long-term negative impact on health-related quality of life, activities of daily living, arm function, and participation for both stroke survivors and their families (Ytterberg, Dybäck, Bergström, Guidetti, \& Eriksson, 2017). The annual cost of treating stroke in the Canadian health care system is about $\$ 4$ billion, including direct and indirect costs (Foundation, 2014). Many of these costs are from rehabilitation (Mittman N, 2012).

Evidence suggests that home-based telerehabilitation (TR) is a viable approach for upper limb training post-stroke when rehabilitation services are not available (Langan, 2013). TR for stroke survivors has emerged as a promising 
intervention for improving motor function, cognitive function, and neuropsychiatric impacts resulting from stroke. The use of telerehabilitation may optimize compliance with treatment through the use of games to increase desirability and promote participation (Baranowski, Buday, Thompson, \& Baranowski, 2008). Travel distance and time are also barriers to accessing rehabilitation treatments and TR fills a need to ensure treatment adherence for those who live in rural areas or live in areas far away from where rehabilitation infrastructures are located (Peretti, Amenta, Khosrow Tayebati, \& Nittari, 2017).

A recent systematic review showed that TR interventions have either better or equal beneficial effects on motor, higher cortical, and mood disorders compared with conventional in-person therapy visits (Sarfo, Ulasavets, Opare-Sem, \& Ovbiagele, 2018). Another systematic review and meta-analysis showed that stroke survivors who use TR achieve comparable levels of daily life activities and health-related quality of life (HRQoL) to those who receive a face-to-face rehabilitation intervention. Moreover, few studies have assessed the cost-effectiveness of the intervention (Tchero, Maturin, Lannuzel, \& Rusch, 2018).

A study conducted by Llorens (2015) showed lower cost for the telerehabilitation program compared to the in-clinic treatment (US\$654). In many telerehabilitation studies the reduced costs are mostly related to travel costs being eliminated for the telerehabilitation group (Thaker, Monypenny, Olver, \& Sabesan, 2013). To control the costs of managing the health consequences of a stroke, it is necessary to evaluate the cost of alternative methods of providing therapy.

Despite the growing literature on the use of telerehabilitation and virtual reality for stroke rehabilitation, few studies have conducted costing analyses. To our knowledge, no studies have estimated the cost of store-and-forward or off-line monitoring of TR-based exercise programs for stroke survivors. This study calculated the cost of a novel, home-based tailored TR program using an affordable virtual reality system for upper-limb rehabilitation post-stroke compared to the current standard of care. A cost analysis of the incremental costs of the telerehabilitation program was conducted and extrapolated to variations of the pilot program studied in this paper. A detailed breakdown of the costs allows providers and researchers to estimate the costs for variations of the program studied here and to tailor the program to their situation.

In times of restricted resources and given the large economic impact of stroke, there is great interest in deploying innovative and alternative stroke care to provide high-quality, affordable therapy that maximizes recovery and ensures efficiency and sustainability of care. This paper provides decision makers a framework for costing the introduction of a TR program and highlights the main issues to consider when starting a program.

\section{Method}

\subsection{The Pilot Project}

A costing analysis was conducted alongside a randomized pilot clinical trial. Participants for the pilot project were recruited from several rehabilitation and community centers in Montreal, Canada. The criteria for inclusion were: (1) ischemic or hemorrhagic stroke (did not have to be a first-time stroke); (2) mild to moderate upper limb impairment (score 3-6 Chedoke-McMaster); and (3) no longer receiving rehabilitation services. Participants who were limited in their participation or understanding of instructions, as determined by their treating therapist, doctor, a caregiver, or themselves were excluded from the study. After considering the inclusion and exclusion criteria, 51 people who had sustained a stroke were recruited and randomly allocated to either the intervention $(\mathrm{N}=26)$ or the control $(\mathrm{N}=25)$ group.

All participants (both the control and the treatment group) were provided with a written home exercise program during an initial appointment with a physiotherapist, as proposed by the Graded Repetitive Arm Supplementary Program (GRASP), tailored to their level, as per current stroke guidelines (Lindsay M, 2010). Guidelines provided by the Canadian Best Practice Recommendations for Stroke Care include the provision of an exercise program for stroke patients with loss of upper limb function. Participants in both the control and the treatment group were asked to perform their exercises at least 5 times per week for 30 minutes a day, for 4 weeks. For participants in the control group, there were no additional visits to the physiotherapist.

In addition to the written exercise program, participants in the treatment group received a TR platform (Jintronix system) (Jintronix) for upper limb rehabilitation using virtual reality (VR). Participants were introduced to the system during the initial appointment with a physiotherapist, during which the at-home exercise program was designed for the participant. Participant performance was logged by the TR platform and monitored off-line by the therapist. Patients were enrolled in the pilot study for four weeks. Over the two-year pilot study, twenty-six patients participated in the TR program 


\subsection{Costing Analysis}

The extra cost of providing the TR program (treatment) compared to just providing the exercise program (control group) was calculated. The only difference between the control and treatment groups was the presence of a TR environment. Both groups had an initial appointment with a physiotherapist and were provided with an exercise program. The cost of providing the exercise program at the initial appointment was assumed to be the same for the control and the treatment groups and was thus not included in the analysis. Patients in both groups were expected to spend the same amount of time participating in the program; therefore, the difference in time costs for the patient between the intervention and control programs was minimal. The introduction of the TR program in this study involved additional costs because the recommended standard of care was received by both the treatment and control groups. The treatment group was also supplied with extra resources to participate in the TR environment which allowed participants to receive a higher intensity of treatment as recommended by the stroke rehabilitation guidelines.

The costing analysis adopted a societal perspective for evaluating the program, a common approach when assessing health programs (Jonsson, 2009). Adopting this viewpoint required that the costs to all parties affected by the program, for example, both health care providers and patients, be measured and valued. As there are no costs borne by a third party due to the intervention, and costs to patients are identical for the treatment and control groups, by measuring the costs of the health authority, the costs were estimated from a societal perspective. All costs were measured in 2015 Canadian dollars and based on 2015 prices.

The cost of the pilot project was calculated first. The fixed costs, that is, the costs to set up the pilot project, were separated from the variable costs, that is, the costs incurred for each patient. Costs for a variation of the pilot project were presented to provide context for the estimated costs.

Finally, the costs of the pilot program were extrapolated to a scenario with a permanently established program with the required hardware in continuous use. Computer equipment lasts longer than a two-year study period and it was useful to consider the cost as spread out over the life of the equipment. Fixed costs were expressed as an annuity, that is, a cost in each year of the expected useful life of the equipment; when the stream of costs was discounted to the current period the sum was equal to the fixed costs of the TR program. Several assumptions must be made to calculate this value. First, the depreciation of the equipment and replacement date had to be estimated. The Canada Revenue Agency allows businesses to depreciate computer equipment at 55\% per year (Canada G. o., 2019), which implied a useful life of 4 to 5 years for the equipment. Second, a discount rate had to be chosen to account for the occurrence of costs at different times. One view of the discount rate was to consider it as adjusting for lost interest. Money spent today could have been invested and yielded a return in the future, so the costs needed to be adjusted for this lost opportunity cost. The Treasury Board of Canada Secretariat currently recommends a discount rate of $8 \%$ for programs with an investment emphasis. For programs with an emphasis on providing consumer consumption, such as health programs, a social discount rate was often used, which was estimated at $3 \%$ for Canada (Canada G. T., 2007).

\section{Results}

\subsection{Cost Analysis of Pilot Project}

The fixed costs of the pilot project were presented in Table 1. The required hardware included a computer, screen, keyboard, mouse, Internet connection, and the Kinect camera. The computer required sufficient CPU power and RAM to support the gaming environment of the software. A screen of at least 27" allowed patients to interact with the software. The Kinect camera recorded the patient's movements for the software and required a Microsoft cable adapter for proper interaction with the Windows environment. These specifications ensured that the TR program functioned properly. The total fixed costs for the program were $\$ 7,765.44$ ( $\$ 298.67$ per patient) for the 26 patients enrolled in the pilot project. 
Table 1. Fixed Costs for Pilot Project

\begin{tabular}{llll}
\hline & Unit cost & Quantity & Total cost \\
\hline Computer & $1,050.00$ & 4 & $4,200.00$ \\
27 " screen & 250.00 & 4 & $1,000.00$ \\
Keyboard + mouse & 30.00 & 4 & 120.00 \\
USB Wifi adapter & 211.36 & 4 & 845.44 \\
Kinect camera (including the Microsoft cable adapter) & 400.00 & 4 & $1,600.00$ \\
Total costs & & $7,765.44$ \\
Cost per Patient in Treatment & & 298.67 \\
\hline
\end{tabular}

The per patient variable costs of the pilot project are presented in Table 2. Total variable costs were $\$ 10,976.94$ ( $\$ 422.19$ per patient). The variable costs were separated into installation/removal costs and costs incurred during participation in the program.

Patients were provided with an Internet key card. The cost of the key card was the price charged by the local telecommunications company. A technician set up the necesssary equipment at the patient's home and recorded the time spent setting up the equipment, transit time to the patient's residence, and the mileage to the patient's residence. The same information was collected when the technicians returned to pick up the equipment. The cost of the technician's time was estimated to be equal to the hourly wage plus benefits. Benefits were estimated to be $10 \%$ of employer's total payroll costs (Stewart, 2015). The cost of mileage was set at $\$ 0.43$ per kilometer as per the hospital's allowed expenses. The average time for installation and removal was 3.31 hours. The average travel distance for installation and removal was $78.69 \mathrm{~km}$.

The variable costs of the pilot project also included technician time spent assisting patients with the TR system, software rental, and the time spent by physiotherapists monitoring patients. Of the 26 patients in the study, only 5 required assistance with the equipment during the program duration. The calculation of the costs of technician time and mileage to provide the assistance was the same as the calculation for installing and uninstalling the equipment. The average time (over the 26 patients in the study) for assistance during the program was 0.42 hours and the average mileage was 3.00. Each patient also required a monthly subscription to the software for the duration of the program, which cost $\$ 25 /$ month. Finally, the costs of patient monitoring by the physiotherapists were included. The physiotherapists recorded the time spent monitoring their patients during the study. On average, they spent 75 minutes monitoring each patient over the 4-week period. The physiotherapist time was valued as the average wage of the physiotherapists monitoring the patients plus an estimate of benefits, which came to $\$ 39.83$ per hour. The average time spent by the physiotherapist reviewing patients' activity on-line and revising exercises was 75 minutes (range $40-150$ minutes) for an average cost of $\$ 49.92$ per patient. The work was conducted on-line so there were no travel expenses.

Table 2. Variable Costs (per patient) for Pilot Project

\begin{tabular}{llll}
\hline & Unit Cost & Quantity & Cost per patient \\
\hline Installation Costs and Removal of Equipment & & & 180.00 \\
Internet Installation cost & 180.00 & 1.00 & 108.30 \\
Installation and Removal of Hardware Time (hours) & 32.72 & 3.31 & 33.84 \\
Installation and Removal of Hardware Mileage (km) & 0.43 & 78.69 & 13.84 \\
\hline Patient Costs During Program & & & 1.29 \\
Hardware Assistance Time (hours) & 32.72 & 0.42 & 10.00 \\
Hardware Assistance Mileage (km) & 0.43 & 3.00 & 25.00 \\
Internet Access (month) & 10.00 & 1.00 & 49.92 \\
Software Rental (month) & 25.00 & 1.00 & 422.19 \\
Physio Time (hours) & 39.83 & 1.25 & \\
Variable Costs per Patient & & & \\
\hline
\end{tabular}




\section{Annualized Cost $=$ Asset Price * $\left(\right.$ Discount Rate $/\left(1-(1+\text { Discount rate })^{\wedge}-\right.$ Periods $)$}

The total incremental cost of the treatment group compared to the control group was $\$ 18,742.38$ (\$720.86 per patient); of which $\$ 7,765.44$ was for fixed costs and $\$ 10,976.94$ was for variable costs.

Patients are likely to have the necessary computer equipment and Internet access, but do not have the Kinetic camera to record their exercise program. Using the detailed information provided above, it was possible to estimate the cost of this variation of the pilot project. The difference in costs between the pilot project and this variation was mainly in the fixed costs of supplying the equipment, as only the camera needs to be provided. For the variable costs, the time required to install and uninstall the equipment is assumed to be the same as in the pilot project. This assumption may underestimate the variable costs of the camera-only program because installing a camera on an unknown system may involve unforeseen difficulties; these were less likely with the pilot project as the same system was being installed for each patient and technicians were familiar with it. In a program that only provides the required camera to patients, total incremental costs would be $\$ 7,636.98$ ( $\$ 293.73$ per patient) compared to total incremental costs for the pilot project of $\$ 18,742.38$ ( $\$ 720.86$ per patient).

\subsection{Cost Analysis of a Permanent Program}

The pilot program treated only 26 patients during the study, which resulted in significant time when the equipment was not being used. We can estimate the incremental costs of a permanent program where the equipment is in continuous use by patients by extrapolating from the cost analysis for the pilot project. It is important to consider this situation because equipment costs were a significant part of the total costs. We extrapolated from the pilot program to estimate the cost for a fully operational program where patients use the equipment for four weeks. Allowing one week for the equipment to be switched between patients, each set of equipment could be used by ten patients in a year. With four sets of equipment available in a fully operational program, forty patients could be enrolled per year.

The annualized fixed costs for the full-equipment and camera-only variations discussed above, for two discount rates (3\% and $8 \%)$ and for both a useful life of 4 and 5 years, are presented in Table 3 . The annualized cost for the full-equipment program ranges from $\$ 1,695$ ( $\$ 42$ per patient) to $\$ 2,344$ ( $\$ 59$ per patient), while for the camera-only variation the range is between $\$ 349$ ( $\$ 9$ per patient) to $\$ 483$ ( $\$ 12$ per patient).

Table 3. Annualized fixed costs

\begin{tabular}{llll}
\hline & Discount Rate & Useful Life & Annualized Value \\
\hline Full-equipment variation & & 4 & \\
$7,892.04$ & 0.03 & 4 & $2,089.11$ \\
& 0.08 & 5 & $2,344.55$ \\
& 0.03 & 5 & $1,695.62$ \\
& 0.08 & & $1,944.90$ \\
\hline Camera-only variation & & 4 & 430.44 \\
$1,600.00$ & 0.03 & 4 & 483.07 \\
& 0.08 & 5 & 349.37 \\
& 0.03 & 5 & 400.73 \\
\hline
\end{tabular}

Table 4 summarizes the total cost of full-equipment and camera-only programs that provide a 1-month program for 40 patients per year. The total costs of the programs are shown using two discount rates $-0.03 \%$ and $0.08 \%$ (a social discount rate and an investment discount rate). The annual variable costs from Table 2 were multiplied by 40 to estimate the total annual costs for a program serving 40 patients per year.

With the higher investment in computer equipment, a full-equipment program was more expensive. An operational program providing care to 40 patients per year would have annual costs between $\$ 19,011$ ( $\$ 475$ per patient) and $\$ 19,271$ ( $\$ 482$ per patient). The camera-only variation would have total costs between $\$ 9,717$ ( $\$ 242$ per patient) and $\$ 9771$ ( $\$ 245$ per patient). 
Table 4. Total Costs of Ongoing program

\begin{tabular}{llc}
\hline & \multicolumn{2}{c}{ Discount Rate } \\
\cline { 3 - 3 } & 0.03 & 0.08 \\
\hline Full Equipment variation & & $2,382.77$ \\
Annualized Fixed Costs & $2,123.17$ & $16,887.57$ \\
Variable Costs & $16,887.57$ & $19,270.34$ \\
Total Costs (A) & $19,010.74$ & 481.76 \\
Total Costs per Patient ((A)/40) & 475.27 & 483.07 \\
\hline Camera-only variation & & $9,287.53$ \\
Annualized Fixed Costs & 430.44 & $9,770.60$ \\
Variable Costs & $9,287.53$ & 244.26 \\
Total Costs (A) & $9,717.97$ & 242.95 \\
Total Costs per Patient ((A)/40) & & \\
\hline
\end{tabular}

\section{Discussion}

This study provides information on a topic with very limited primary data - the intervention costs associated with delivering telerehabilitation and virtual reality in stroke survivors. In addition, the detailed presentation of the costs allows the results to be extrapolated to estimate the costs for different scenarios. The program studied in this paper had patients completing exercises on their own at home and did not require travel for patients.

This study fills a significant gap in the literature related to costs of TR for stroke survivors. A recent systematic review of the topic found no studies measuring the cost effectiveness of TR in stroke survivors. However, evidence was found that TR is less expensive to implement compared to face-to-face rehabilitation in other patient populations. Furthermore, there is evidence that TR improves several outcomes such as balance, mobility, motor recovery, pain reduction and verbal communication skills in several conditions, including stroke (Coulibaly, Rochefort, Kairy, \& Tousignant, 2018). Telerehabilitation is often proposed as an approach to provide rehabilitation interventions when these are not otherwise accessible. Therefore, it is important to have a better understanding of the costs incurred when proposing such interventions to those who would not otherwise have access to them. This study provides an alternate approach to examing costs using an incremental cost analysis that can contribute to the decision making process when introducing telerehabilitation.

Our study has limitations that should be taken into account. Assumptions about the discount rate must be made when costs occur across multiple periods. The preferred discount rate may vary between organizations and the information is provided so that different discount rates can be applied. Similarly, costs could be region-specific because of differences in the market or the scale of the proposed project, thereby requiring the per unit price to be adjusted. The prices used to calculate the cost of technology are from the pilot project; however, the cost of technology constantly changes and these prices have likely changed over time. A final limitation is that a cost-benefit, or cost-effectiveness, analysis using the efficacy findings has not been conducted because the preliminary effectiveness results of the intervention are not finalized. Including information about the benefits of using the TR environment would allow decision-makers to decide if the program was worth introducing.

\section{Conclusion}

This cost-analysis study will facilitate the implementation and future research on cost-effectiveness on TR interventions. The objective of this study was to calculate the additional cost of a short-term telerehabilitation and virtual reality intervention for improving upper limb function in chronic stroke survivors and estimate the cost of different variations of the program. Despite the growing literature on the use of telerehabilitation and virtual reality for stroke rehabilitation, few studies have conducted costing analyses on stroke rehabilitation. After calculating the costs for the pilot project that was the basis for this study, we extrapolated the results to consider a variation of the project and the cost for a permanent program. The findings of this study provide background information for the potential implementation of telerehabilitation for stroke survivors, to improve accessibility to rehabilitation services.

The information gathered in this study could inform decision making and clarify necessary steps to promote 
effective implementation of the telerehabilitation and virtual reality intervention for stroke survivors, thereby improving accessibility to rehabilitation services and outcomes post stroke. In addition, it could potentially be used to help decision-makers tailor implementation cost strategies for telerehabilitation interventions. It is expected that the information in this study will guide and facilitate the planning and implementation of telerehabilitation interventions for stroke survivors.

\section{Acknowledgments}

This research was funded by The Heart and Stroke Foundation of Canada. Mirella Veras, Dahlia Kairy and Jennifer Stewart contributed to research conception/design, data acquisition, data analysis/interpretation, manuscript preparation and final approval. Raywat Deonandan contributed to research conception/design, data analysis/interpretation, manuscript preparation and final approval. José CarlosTatmatsu-Rocha, Johanne Higgins and Lise Poissant contributed to data analysis/interpretation, manuscript preparation and final approval. Dahlia Kairy was the principal investigator.

Our thanks to Alejandro Hernandez for his support throughout the study.

\section{Competing Interests Statement}

The authors declare that there are no competing or potential conflicts of interest.

\section{References}

Baranowski, T., Buday, R., Thompson, D., \& Baranowski, J. (2008). Playing for real: video games and stories for health-related behavior change. American journal of preventive medicine, 34(1), 74-82. https://doi.org/10.1016/j.amepre.2007.09.027

Baumann, M., Le Bihan, E., Chau, K., \& Chau, N. ( 2014). Associations between quality of life and socioeconomic factors, functional impairments and dissatisfaction with received information and home-care services among survivors living at home two years after stroke onset. BMC Neurology, 14, 92, 1-12. https://doi.org/10.1186/1471-2377-14-92

Canada, Government. $\quad$ (2019). Costs. $\quad$ Retrieved from https://www.canada.ca/en/revenue-agency/services/tax/businesses/topics/sole-proprietorships-partnerships/r eport-business-income-expenses/claiming-capital-cost-allowance/classes-depreciable-property.html\#class50

Canada, G. T. (2007). Canadian Cost-Benefit Analysis Guide: Regulatory Proposals. Retrieved from https://www.tbs-sct.gc.ca/rtrap-parfa/analys/analys-eng.pdf

Coulibaly, L. P., Rochefort, C., Kairy, D., \& Tousignant, M. (2018). Cost-effectiveness analysis of telerehabilitation for people who have sustained a stroke with return home without intensive rehabilitation: A systematic review. International Journal of Hospital-Based Health Technology Assessment, 1, 19-44.

Foundation., H. (2014). $2013 \quad$ Stroke Report. Retrieved from http://www.heartandstroke.com/atf/cf/\%7B99452D8B-E7F1-4BD6-A57D-B136CE6C95BF\%7D/StrokeRe port2013_ENG.pdf

Jintronix. (n.d.). Jintronix Rehabilitation System. Retrieved 17 May, 2019, from http://www.jintronix.com/.

Jonsson, B. (2009). Ten arguments for a societal perspective in the economic evaluation of medical innovations. The European Journal of Health Economics, 10, 357. https://doi.org/10.1007/s10198-009-0173-2

Kitzmüller, G., Asplund, K., \& Häggström, T. (2014). The Long-Term Experience of Family Life After Stroke. Journal of Neuroscience Nursing, 44(1). .

Krpic, A., Savanovic, A., \& Cikajlo, I. (2013). Telerehabilitation: remote multimedia-supported assistance and mobile monitoring of balance training outcomes can facilitate the clinical staff's effort. Int J Rehabil Res, 36, 162-71. https://doi.org/10.1097/MRR.0b013e32835dd63b

Langan, J. D. (2013). Home-based telerehabilitation shows improved upper limb function in adults with chronic stroke: a pilot study. Journal of rehabilitation medicine, 45(2), 217-220. https://doi.org/10.2340/16501977-1115

Laver, K., George, S., Thomas, S., Deutsch, J., \& Crotty, M. (2011). Virtual reality for stroke rehabilitation. Cochrane Database Syst Rev, (9), CD008349. https://doi.org/10.1002/14651858.CD008349.pub2

Lindsay, M. G. G. (2010). Canadian Best Practice Recommendations for stroke care (Update 2010). On behalf of the Canadian Stroke Strategy. Best Practices and Standards Writing Group. Ottawa, Ontario Canada: Canadian Stroke Network. 
Lloréns, R. N. E. (2015). Effectiveness, usability, and cost-benefit of a virtual reality-based telerehabilitation program for balance recovery after stroke: a randomized controlled trial. Arch Phys Med Rehabil. Mar, 96(3), 418-425. https://doi.org/10.1016/j.apmr.2014.10.019

Mittman, N. S. S. (2012). Impact of disability status on ischemic stroke costs in Canada in the first year. Canadian Journal Neurology Science, 39(6), 793-800. https://doi.org/10.1017/S0317167100015638

Nichols-Larsen, D., Clark, P., Zeringue, A., Greenspan, A., \& Blanton, S. (2005). Factors influencing stroke survivors' quality of life during subacute recovery. Stroke, 36(7), 1480-4. https://doi.org/10.1161/01.STR.0000170706.13595.4f

Peretti, A., Amenta, F., Khosrow Tayebati, S., Nittari, G., \& Sarosh Mahdi, S. (2017) Telerehabilitation: Review of the State-of-the-Art and Areas of Application. JMIR Rehabil Assist Technol., 4(2), e7. https://doi.org/10.2196/rehab.7511

Roberto, L., Enrique, N., Carolina, C., \& Mariano, A. (2015). Effectiveness, Usability, and Cost-Benefit of a Virtual RealityeBased Telerehabilitation Program for Balance Recovery After Stroke: A Randomized Controlled Trial. Archives of Physical Medicine and Rehabilitation, 96, 418-25. https://doi.org/10.1016/j.apmr.2014.10.019

Salter, K. F. N. (2007). Assessment of participation outcomes in randomized controlled trials of stroke rehabilitation interventions. Int J Rehabil Res, 30, 339-42. https://doi.org/10.1097/MRR.0b013e3282f144b7

Sarfo, F., Ulasavets, U., Opare-Sem, O., \& Ovbiagele, B. (2018). Tele-Rehabilitation after Stroke: An Updated Systematic Review of the Literature. $J$ Stroke Cerebrovasc Dis., 27(9), 2306-2318. https://doi.org/10.1016/j.jstrokecerebrovasdis.2018.05.013

Stewart, N. (2015). Benefits Benchmarking 2015. Ottawa: The Conference Board of Canada.

Tchero, H., Maturin, T., Lannuzel, A., \& Rusch, E. (2018). Telerehabilitation for Stroke Survivors: Systematic Review and Meta-Analysis. J Med Internet Res., 20(10), e10867. https://doi.org/10.2196/10867

Tessier, A. l'Institut national d'excellence en santé et en services sociaux (INESSS). (2012). L'organisation et la prestation de services de réadaptation pour les personnes ayant subi un accident vasculaire cérébral (AVC) et leurs proches. Rapport rédigé par Annie Tessier.

Thaker, D., Monypenny, R., Olver, I., \& Sabesan, S. (2013). Cost savings from a telemedicine model of care in Northern Queensland, Australia. Med J. Aust., 199(6), 414-417. https://doi.org/10.5694/mja12.11781

Ytterberg, C., Dybäck, M., Bergström, A., Guidetti, S., \& Eriksson, G. (2017). Perceived impact of stroke six years after onset, and changes in impact between one and six years. J Rehabil Med., 49(8), 6, 37-643. https://doi.org/10.1037/0033-2909.126.6.910

\section{Copyrights}

Copyright for this article is retained by the author(s), with first publication rights granted to the journal.

This is an open-access article distributed under the terms and conditions of the Creative Commons Attribution license (http://creativecommons.org/licenses/by/4.0/). 